\title{
Modelling Workflow for Segmented Timber Shells Using Wood-Wood Connections
}

\author{
Petras Vestartas ${ }^{(\bowtie)}$, Nicolas Rogeau, Julien Gamerro, \\ and Yves Weinand \\ EPFL ENAC IIC IBOIS, GC H2 711, 1015 Lausanne, Switzerland \\ petras.vestartas@epfl.ch
}

\begin{abstract}
This paper presents a modelling method based on planarization for double-curved segmented timber shell made from quad polygons and assembled by wood-wood connections. The inspiration is taken from timber dome structures $[1,2]$, where solid timber walls were built from planks, connected side by side. Furthermore, the research is based on a collaboration with a local timber company located in a mountain area. In this context, timber has a low economic value because the price no longer covers the harvesting costs [3]. Therefore, there is a need to explore the available timber stock (round wood, beam elements and planks) to transform it locally. The geometry modelling workflow is split into three parts: surface discretization, joint modelling and fabrication. Firstly, projection-based solver is applied to the planarization of volumetric blocks. Secondly, the joint geometry is computed according to the insertion vector and a tool-path is generated using G-Code [4] to guide the 4.5 Axis CNC machining. As proof of concept, two prototypes were built, one from planks and another from round-woods. The choice of material influenced the segmentation of the timber shell. Finger and Tenon-mortise joinery techniques have been chosen for their simple modelization and fast cutting time. Their placement follows as closely as possible fiber orientation of wood. Even if both study cases share the same discretization method, the first prototype from timber plates takes advantage of lightweight structures, while the second explores a heavy solid round-wood structural system.
\end{abstract}

Keywords: Discrete polygons $\cdot$ Planarization $\cdot$ Segmentation $\cdot$ Timber shell $\cdot$ Wood-wood connections

\section{Introduction}

\subsection{Research Interest - Wood from Mountain Timber Forestry}

The research project is related to a larger mountain forestry study (tree species - Épicéa), CNC fabrication tool and wood-wood connection development within a CAD interface (see Fig. 2A1-A5). Economically, local mountain forestry no longer covers operating costs due to complicated harvesting process: a crane and a cable system is needed to carry tree trunks from high to low altitude of mountains and then transported them to large sawmills far from a cutting area. To reduce this expensive cycle, there is a preference to investigate novel building systems made from harvested trees and apply them locally. 
(a)

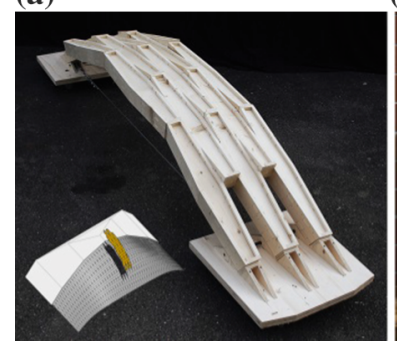

(b)

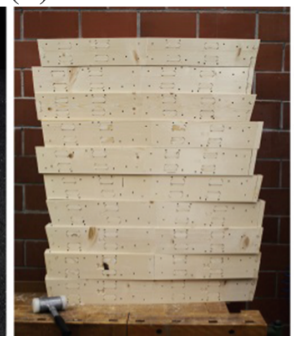

(c)

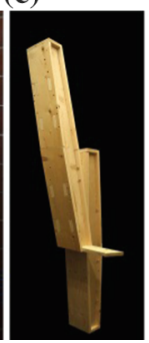

(d)

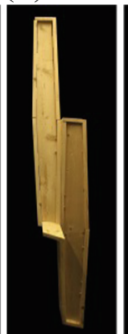

(e)

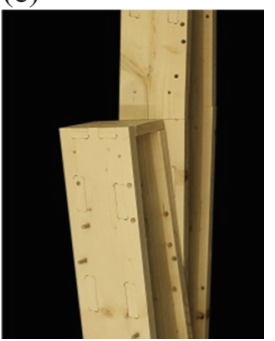

Fig. 1. (A) The prototype from planks, (B) Box components (C-D-E) Connections - dowels

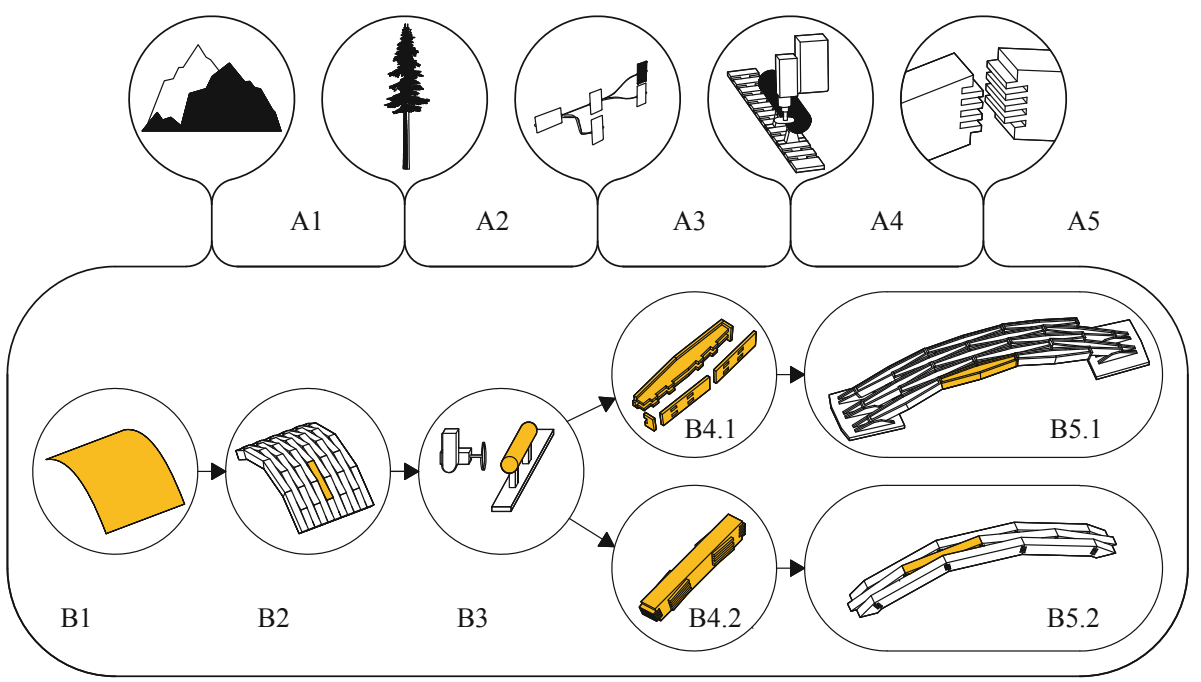

Fig. 2. The research context: A1 - Mountain forestry, A2 - Tree species - Épicéa, A3 - CAD interface, A4 - CNC cutting, A5 - joinery. Modelling workflow: B1 - surface, B2 - discretization and planarization, B3 - CNC milling and cutting, B4.1/2 Box component, Solid element component B5.1/2 Prototypes.

\subsection{Modelling Framework}

The CAD modelling workflow (see Fig. 2B1-B5) is based on a surface subdivision, discretization, planarization, joinery geometrical specification, $\mathrm{CNC}$ fabrication and assembly sequence. Two building materials are assessed from the local saw mill: planks and round-wood. The design target is a surface that is discretized according to building material scale and assembled manually using wood-wood connections. It considers geometrical and material constraints: linearity of wood, grain direction, drying and fabrication processes. The tool is based on a mesh modelling framework in order to have a low-poly representation of panels and timber blocks. In addition to this, the model itself has two representations: a $3 \mathrm{~d}$ model and a fabrication tool-path. 


\section{Surface Discretization}

The planarization method is based on support structures of polygonal cell packing [5]. The aim is to compute polyhedrons, which are solids with only planar face boundaries from arbitrary surface subdivision [6]. Similar research [7] implemented plane projection methods on quad meshes with small gaps at each mesh vertex. Other possibility is to use Voronoi diagrams for planarization [5]. Another technique is to apply a circle packing of triangle meshes whose incircles form a packing [8]. A third method uses circular and conical meshes [9].

A

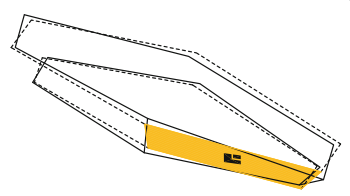

B

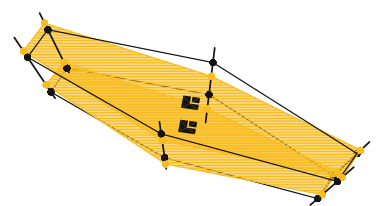

C

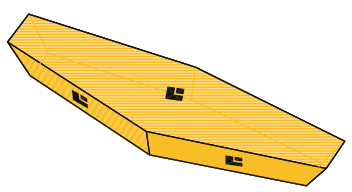

Fig. 3. Planarization method consists of two main steps: (A) planarization of extruded mesh edges (support structure) (B) intersection of lines to average planes of line ends (C) planar polyhedron

A

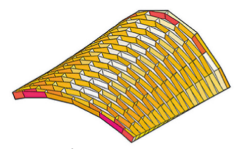

Iterations

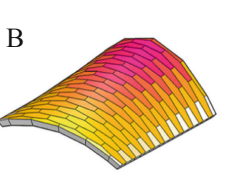

Interval

0.00

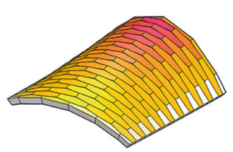

0.25

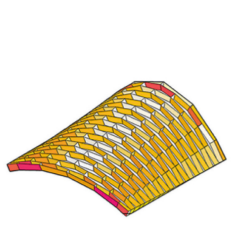

Planarization

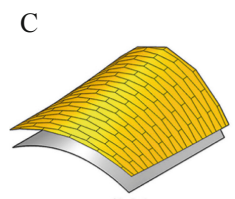

Surface Subdivision
Deviation from Side Plane
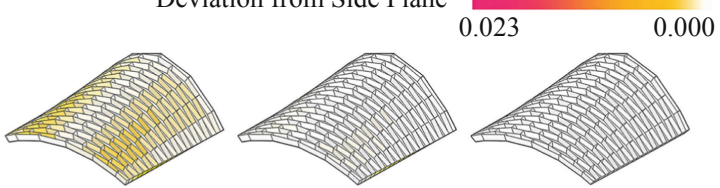

20

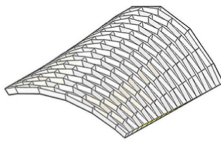

100

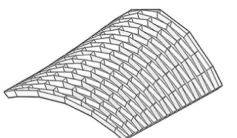

600

Deviation from Top and Bottom Plane
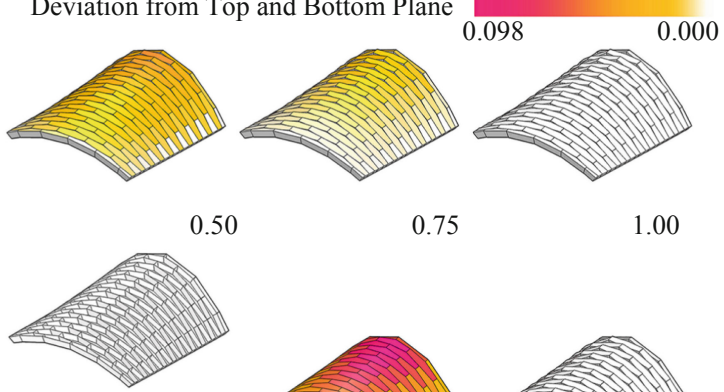

Extruded Edges
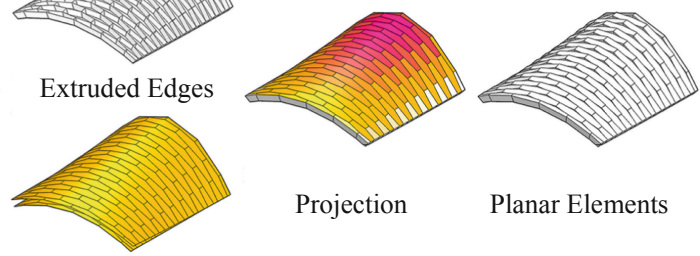

Projection

Planar Elements

Mesh Offset

Fig. 4. Planarization method shown in iterations: (A) planarization of extruded mesh edges 0600 iteration (B) intersection of lines to average planes (C) the full sequence of planarization. 
Our chosen polygonal pattern does not have such qualities because its shape is linked to the linearity of planks and round-wood. The aim is therefore, to keep the original shape without too much distortion. The proposed planarization method is based on an iterative solver which gradually projects extruded quad faces to an average plane (see Fig. 3). A source code of planarization is detailed in Table 1. The user has to specify a tolerance of a planar polygon and give a collection of mesh vertex points. While iterating through polygonal mesh faces, the current mesh face vertices are projected to an average plane and the non-planar polygon deviation is recorded. Afterwards the average sum of projected mesh face vertices are assigned to current mesh face vertices. This method is re-iterated multiple times until the deviation is below the user specified tolerance value.

The full modelling framework is subdivided into the following steps (see Fig. 4C): the target surface is discretized into a polygonal mesh. The mesh edges are extruded and planarized (see Fig. 4A). The top and bottom contours of each cell are not planar and are therefore, projected to the average plane of their vertices (see Fig. 4B). Projected points form a planar polygon and, together with planarized quads, result in a planar polyhedron.

Table 1. Source code of planarization.

\begin{tabular}{lll}
\hline Comment & Source Code & Type \\
\hline $\begin{array}{l}\text { Output } \\
\text { Input }\end{array}$ & $\begin{array}{l}\text { double } \mathrm{d} \\
\text { Point3d[] allPts } \\
\text { Input }\end{array}$ & $\begin{array}{l}\text { Deviation } \\
\text { Point }(\mathrm{x}, \mathrm{y}, \mathrm{z})\end{array}$ \\
& Weights
\end{tabular}




\section{Prototypes}

A series of prototypes was made to validate the planarization algorithm. Two models are detailed in the following Subsect. 3.1 and 3.2. Planks and round-woods were specifically chosen as part of a collaboration between a local forestry company and the research institute to use raw timber materials. Consequently, it was important to understand the relationship between material, connection geometry, assembly sequence and fabrication.

\subsection{Prototype in Planks}

Several existing publications describe the automation of assembly path that influences the joinery geometry (type of joint and an insertion direction) [10-12]. It is also possible to look at this problem from a user perspective when the assembly path is already known. Proposed methodology is based on the existing state-of-the-art method [7] and extended to polygonal meshes, which are not restricted to triangle or quad discretization. Moreover, graph-search methods such as Breadth-First-Search could be applied to compute an assembly order (see Fig. 7). Edges of this graph whose nodes are polygon centers give insertion direction. This vector has to be compared against connected edge direction to know if the insertion vector is not parallel. BFS is a fast approach when a user does not have a sequential order how to assemble elements one after another. Other methods such as a grid-like assembly could be applied when the initial topology is regular. Lastly, several large patches could be assembled together too. It often requires special details, as multiple edges may not have uniform insertion vector applicable to all elements within a group.
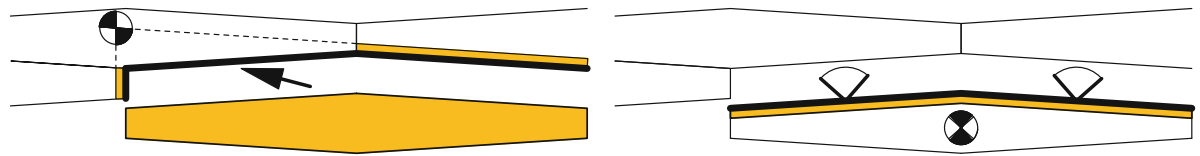

$\mathrm{C}$
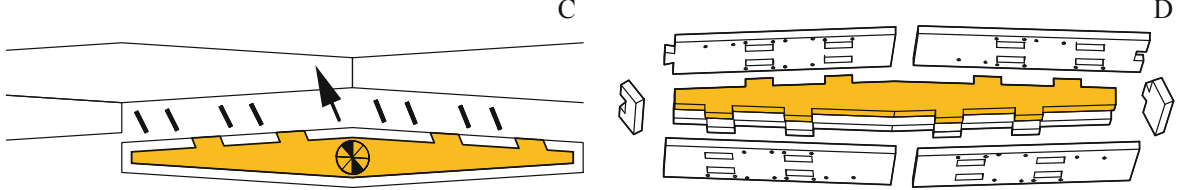

Fig. 5. Computing insertion direction: (A) the insertion range based on neighbor faces (B) the insertion range based on element faces and fabrication constrains (C) intersection between set $\mathrm{A}$ and $\mathrm{B}$ - $\mathrm{A} \cap \mathrm{B},(\mathrm{D})$ - generated geometry.

A series of box-to-plates transformations are made after the planarization. First, we get the insertion range based on neighbor faces (see Fig. 5A) and second, the insertion angle is computer based on element faces and fabrication constrains (a maximum CNC 
cutting angle $45^{\circ}$; see Fig. 5B). Third, we compute the intersection between the two sets of angles $A \cap B$ (see Fig. 5C). Then, turn each polygonal face into a 3D geometry, with plate-to-plate and box-to-box connections. The insertion direction is used to compute tenon-mortise joints at the top and bottom edges. Edge-wise dovetail joints are chosen for each side. To prepare the cutting files, polylines are oriented from the 3D space to the 2D plane of the wooden board. The G-Code is exported for 4, 5 Axis CNC fabrication and when fabrication is finished, individual plates are assembled into boxes. Finally, a set of boxes are connected using dowels (see Fig. 6).

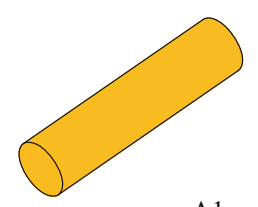

A1

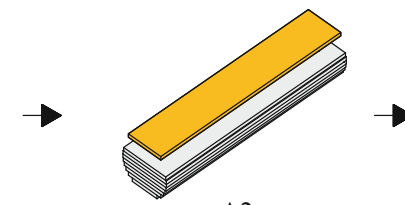

A2

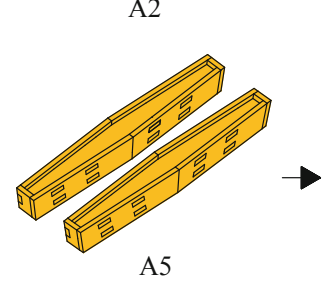

A5

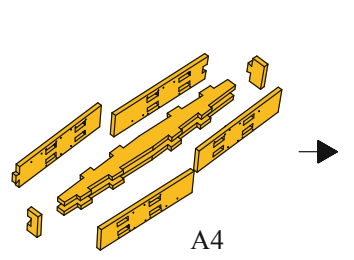

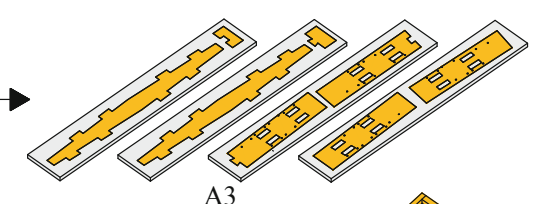

A3

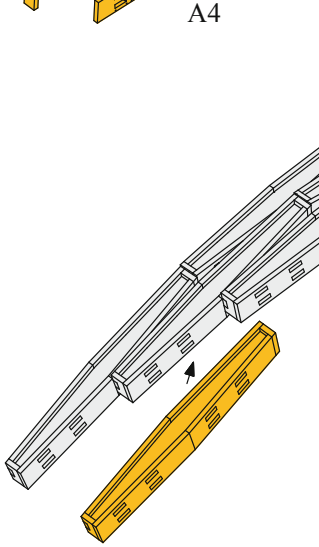

B2

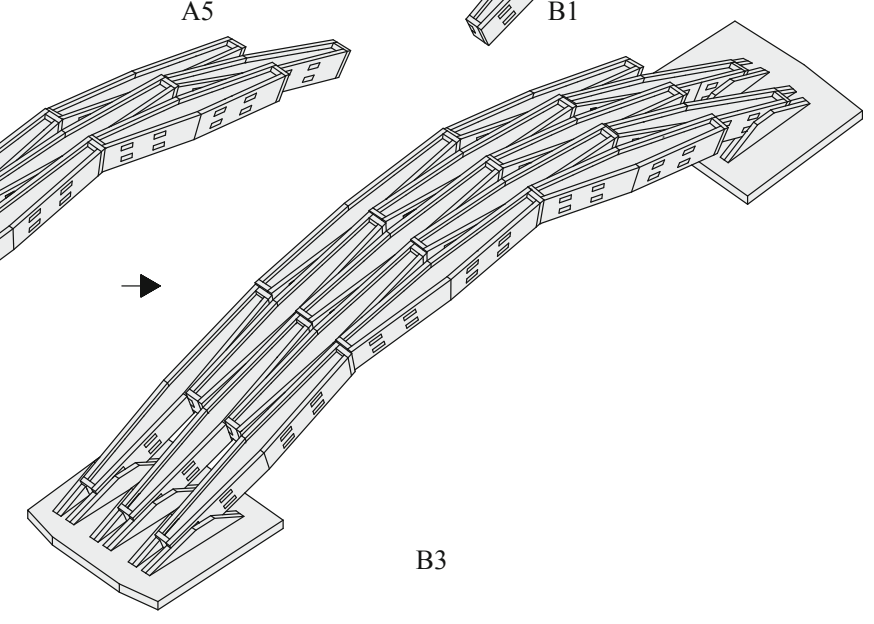

Fig. 6. An assembly sequence: (A1) round-wood, (A2) planks, (A3) cutting outlines, (A4-A5) assembly of a box, (B1) connectng boxes by dowels, (B2) boxes assembly (B3) final prototype. 

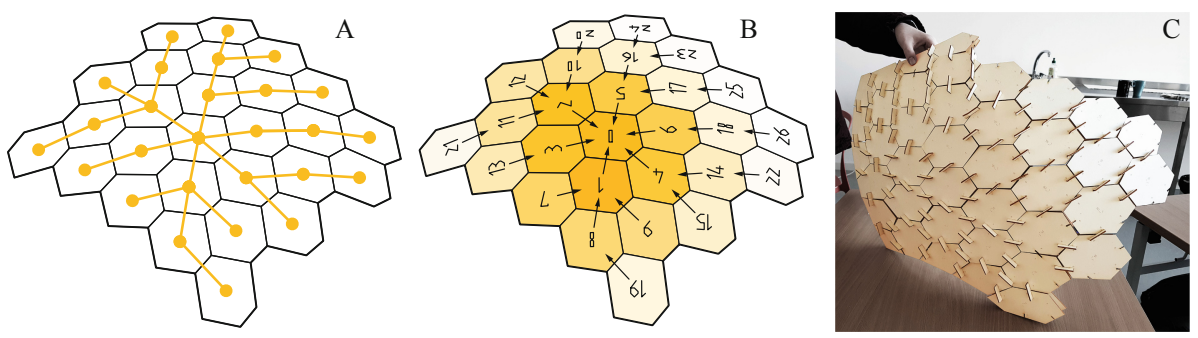

Fig. 7. Graph search algorithm - Breadth-First-Search used for an assembly path and joinery orientation. BFS connectivity graph from center points of hexagons (A), graph edge is used as a vector for each polygon edge joint orientation (B), physical test (Lina Vestarte master's thesis in a collaboration with Petras Vestartas, Romualdas Kučinskas https://www.linavestarte.com/ Assembly-of-Double-curved-Self-supporting-Structure) (2) from cardboard (C).

\subsection{Prototype in Round-Wood}

Another modelling methodology is tested for the Round-Wood prototype. It does not rely on mesh topology but rather on basic geometric transformations and tiling operations for single curved arcs. The planar blocks of the arch are generated using five steps (see Fig. 8). First, curves are divided into points and normal planes are computed from curve derivatives. Two planar outlines are generated using perpendicular planes (see Fig. 8A). Second, a finger joint is drawn as a tile to generate the butt connections for each element. This allows the shape of the connector to be changed any other 2D-joint later. The tile is then oriented from the original plane to the perpendicular frames on the curve (see Fig. 8B). Afterwards, the tile polyline is inserted into the existing planar outline.

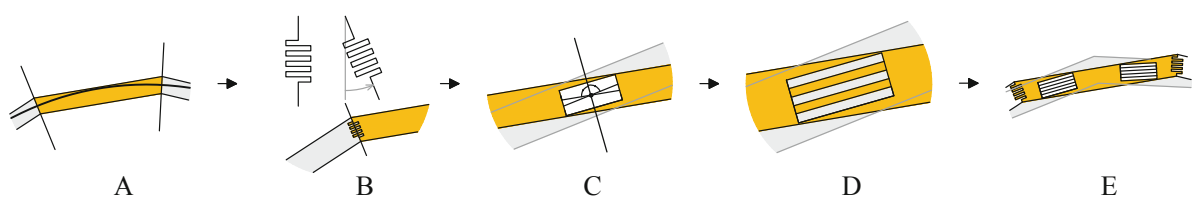

Fig. 8. A sequence of modelling steps: curve interpolation (A), Butt joint (B), intersection area (C), lateral connection (D), full model (E).

Lateral connections are placed using another set of rules. Rectangles are drawn at the intersection of the neutral-axis of each row of elements (see Fig. 8C), the bisector line allowing to maximize the size of the joint placed in the shared area of adjacent timber blocks. Lateral finger joints are generated by subdividing each rectangle and alternately extruding the stripe in and out of the element (see Fig. 8D-E). The divisions within the joints is based on the CNC machining constraints. Finally, a Boolean operation is used to merge lateral joints with the rest of each element. The fabrication method required two different cutting steps: first saw-blade removed large pieces wood and then surfaces were milled using $14 \mathrm{~mm}$ diameter drilling bit (see Figs. 9 and 10). 
A1

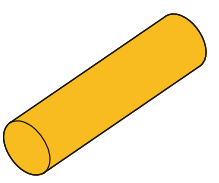

A4

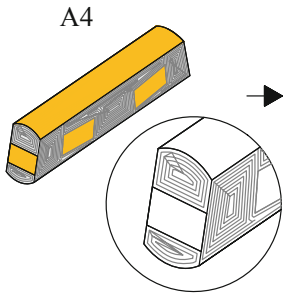

B2
A2

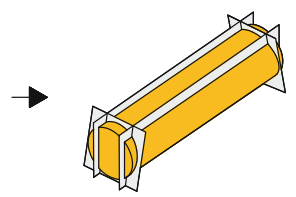

A5

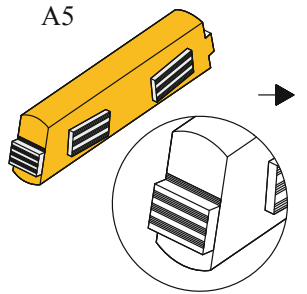

A3

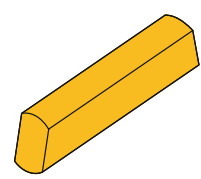

A6
B1

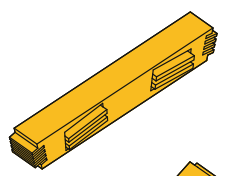

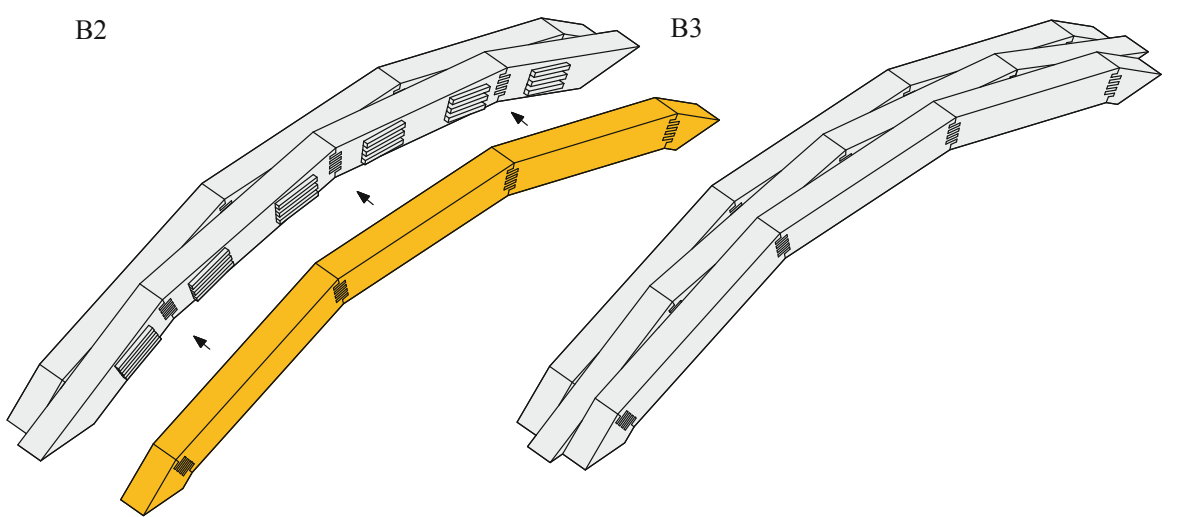

Fig. 9. A sequence of fabrication: (A1) round-wood (A2-A3) cutting planes for a blade saw (A4) milling (A5) cutting grooves (A6) target geometry. Sequence of assembly (B1) Assembly of an arch (B2) Assembly of arch (B3) finished prototype

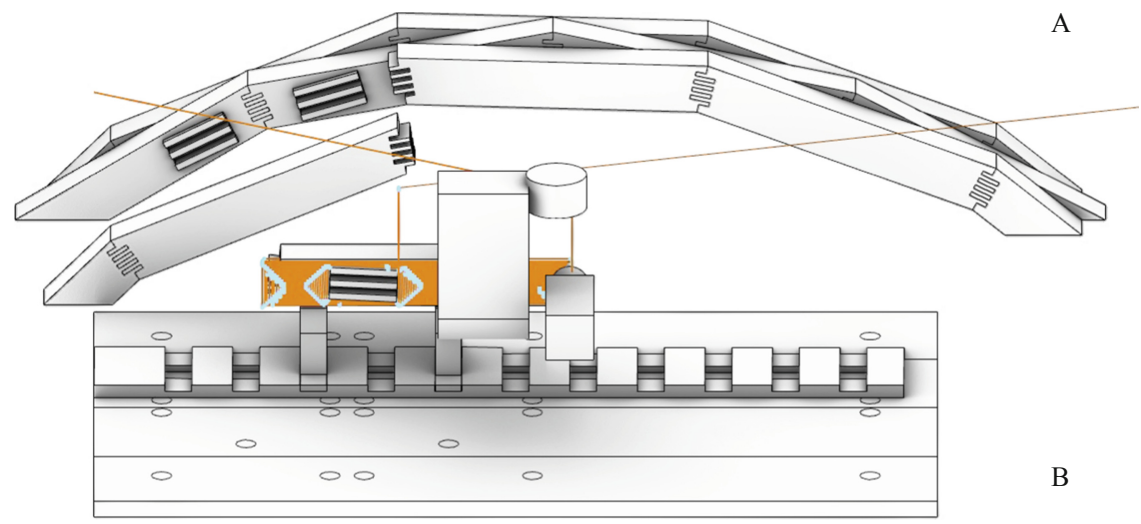

Fig. 10. CNC fabrication of a round-wood specimen. (A) Protype (B) CNC Fabrication. 


\section{Results}

The proposed modelling framework was applied for a series of free-from surfaces ranging from low-resolution and high-curvature (see Fig. 11A-B) to high-resolution and low-curvature geometries (see Fig. 11C-D-E). The shift between polyhedrons is inevitable but acceptable, depending on a Gaussian curvature. In addition, planarized and original polyhedron shapes are similar and do not have large distortions, which makes this workflow useful for our material-based exploration within specific range of surface curvatures.

A

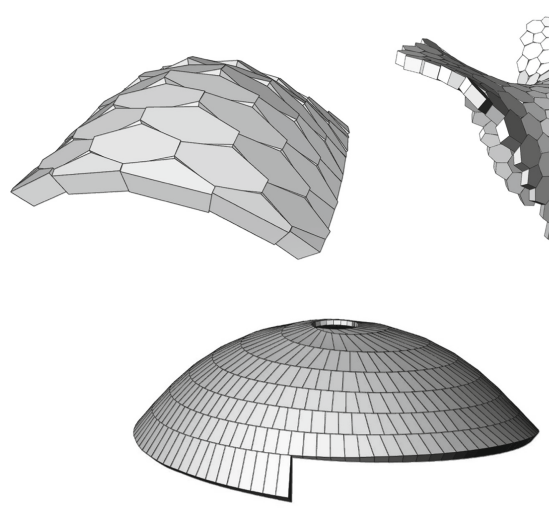

B

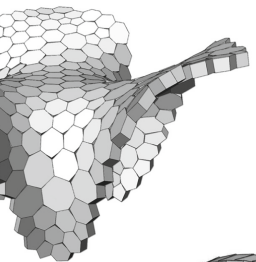

$\mathrm{C}$

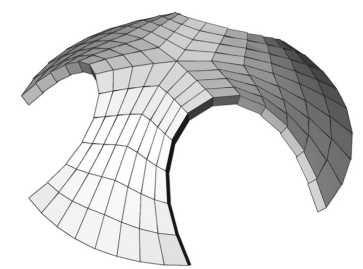

Fig. 11. Planarization method performed on a set of free form shapes (A) low resolution hexagonal subdivision (B-C) high-curvature mesh dual (D) dome (E) low gaussian curvature.

Two prototypes were made using this modelling framework (see Figs. 1, 12, 13). While the underlying geometry of both study cases were similar, we used different CNC fabrication approaches. The plate model was fabricated using CNC cutting while the round-wood specimen required a combination of tools (saw-blade, milling and drilling) and an additional rig for lifting the trunks. Both models were assembled manually with the arch resting on one side so that the elements could be easily assembled on top of each other sequentially. Upon the completion of the assembly the whole specimen was lifted and rotated by $90^{\circ}$ to its intended position.

A geometry mesh library was made for this polygonal surface discretization workflow. It is based on triangle meshes that are grouped to N-Gon representation. The library contains mesh adjacency queries, subdivision, transformation and planarization methods applicable not only for this material-based research. Finally, a G-Code library was made to translate the $\mathrm{CAD}$ geometry to $\mathrm{CNC}$ tool-path such as two open-polyline cutting, surfacing, u-shape saw-blade cutting and collision detection simulation. The tool-path was verified by fabrication tests to avoid damaging tools or de-calibration. 


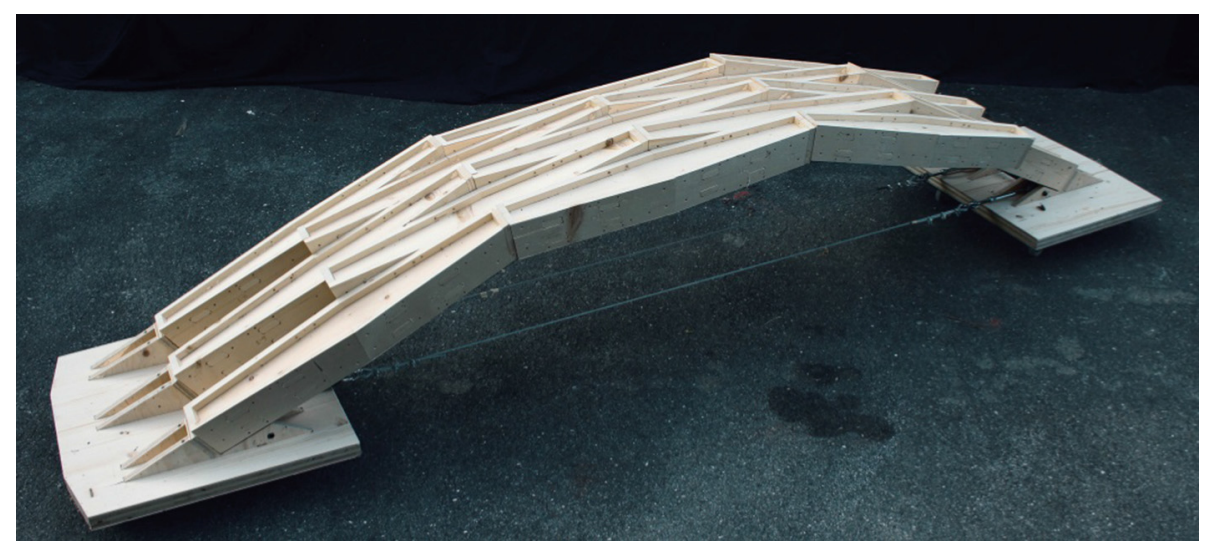

Fig. 12. Prototype from planks.

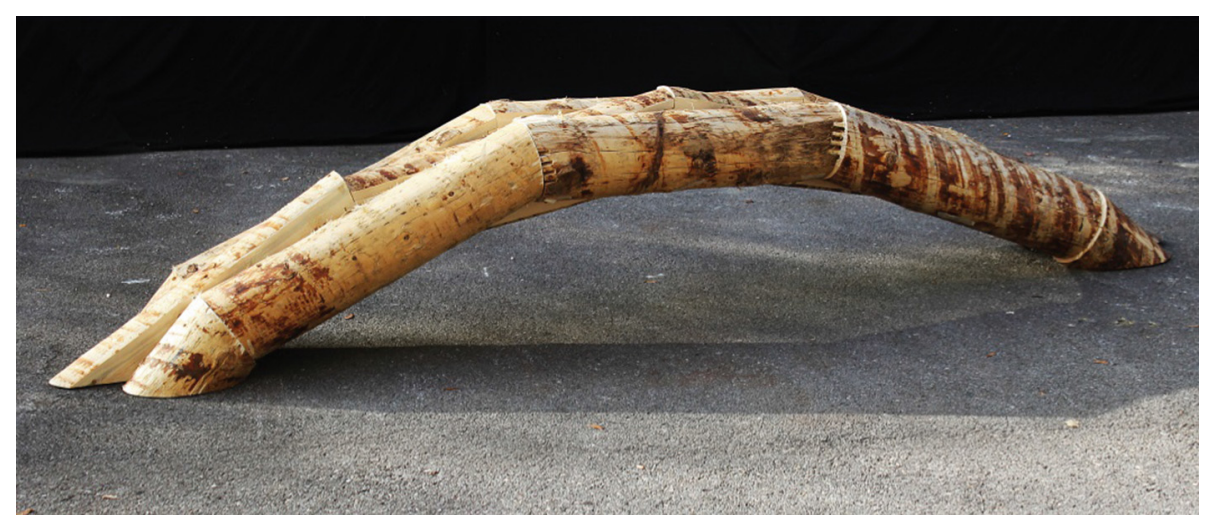

Fig. 13. Prototype from round-woods.

\section{Challenges in Scaling}

Currently the research is focused on an industrial transfer of a full-scale building application. The project includes a free-form roof made from hexagonal components which was accepted by local municipality (see Fig. 14). Several geometrical and structural issues have to be addressed for the future development: assembly methods such as element by element, arch by arch or clustering, roof cladding based on the local carpentry methods, facade detailing, supports. Since the study is currently performed in a research environment, the methodology needs to be transferred to the local fabricator. The material characterization for the spruce is performed by non-destructive testing for $1 \mathrm{~m}^{3}$ of planks but further destructive analysis is needed. In addition to this, external industrial partner ${ }^{1}$ demonstrates the applicability of the shell-like structural systems

\footnotetext{
${ }^{1}$ Imax-Pro, Mobic, https://www.imaxpro.be/fr/realisation-de-double-courbes-bois/.
} 
within robotic fabrication framework that has been recently implemented for the research scope.

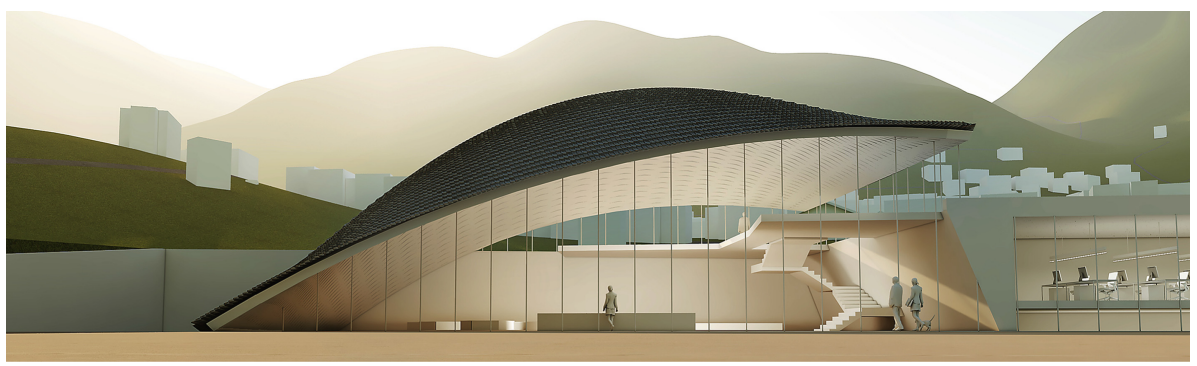

Fig. 14. Concept model for a larger application.

\section{Conclusion}

The present study shows a series of experiments based on a vault discretization. The aim of this workflow is to consider the actual shape of local wood stock, the discretization of a design target, the assembly sequence, joinery generation, 3D model representation and tool-path generation. In addition, we avoided an interoperability issue when two models were successfully executed within one software framework from graphical representation to CNC cutting. The physical model, that was made from planks has a fluent setup when the $3 \mathrm{~d}$ model and the tool-path are modelled equally by a pair of polylines, while the round-wood prototype took more time to prepare the cutting tool-path, thus require more investigation.

There is a series of sub-topics that could be addressed in a more detail such as joinery, assembly logic (BFS), tool-path development and the collaboration framework with industrial partner for a full scale application on Sect. 5. The assembly logic and configuration of joinery are higly interlinked, since the joint orientation follows the direction of insertion. Also, larger assemblies have to be taken into account to understand how clusters of components could be assembled and what connection methods could be applied at boundary condition.

The most constrained part when working with timber from local forests is that it is strongly direction dependent. It requires specifying the wood joinery according to the fiber direction which limits the geometry exploration within the surface subdivision approach. We are aware that timber in its natural form points to timber frame assemblies when joints are positioned at each end of a beam, elsewise additional connectors are required such as screws, dowels or keys.

The global scope is the development of a methodology based on a performative use of the raw sawn timber available in the local forestry to increase the use of timber biomass. The economical consideration is related to forest investigation that shapes and defines the input library for the modelling workflow. It contributes as a study case based on a material given by the research partner. It is an economical and sustainable reasoning where geometrical exploration is applied for a combined chain value. 


\section{References}

1. Philibert, D.: Illustrations de Nouvelles inventions pour bien bastir et a petits fraiz 1561

2. Barbara, M.: Wooden Domes - History and Modern Times-Springer 2018. Springer, Cham (2018)

3. Luthi, S., Remy, C., Miklos, I.: Plan de Gestion des Forets Communales de Rossiniere. $4^{\text {eme }}$ arrondissement forestier, Canton de Vaud (2008)

4. ISO 6983-1:2009: Automation systems and integration-Numerical control of machinesProgram format and definitions of address words - Part 1: Data format for positioning, line motion and contouring control systems

5. Pottmann, H., Jiang, C., Hobinger, M., Wang, J., Bompas, P., Wallner, J.: Cell packing structures. Comput.-Aided Des. (2015). https://doi.org/10.1016/j.cad.2014.02.009

6. Rippmann, M., Van Mele, T., Popescu, M., Augustynowicz, E., Echenagucia, T.M., Barentin, C.C., Frick, U., Block, P.: The armadillo vault computational design and digital fabrication of a freeform stone shell. In: Advances in Architectural Geometry, pp. 344-363 (2016). https://doi.org/10.3218/3778-4_23

7. Robeller, C., Konakovic, M., Dedijer, M., Pauly, M., Weinand, Y.: A double-layered timber plate shell - computational methods for assembly, prefabrication, and structural design. In: Advances in Architectural Geometry (2016). https://doi.org/10.3218/3778-4_9

8. Schiftner, A., Hobinger, M., Wallner, J., Pottman, H.: Packing circles and sphere on surfaces. In: SIGGRAPH Asia 2009 Papers Article No. 139 (2009)

9. Liu, Y., Pottmann, H., Wallner, J., Yang, Y.-L., Wang, W.: Geometric modeling with conical meshes and developable surfaces. ACM Trans. Graph. 25(3), 681-689 (2006). ACM SIGGRAPH

10. Wang, Z., Song, P., Pauly, M.: DESIA: a general framework for designing interlocking assemblies. ACM Trans. Graph. (2018). (Proceedings of SIGGRAPH Asia)

11. Yao, J.X., Kaufman, D.M., Gingold, Y., Agrawala, M.: Interactive design and stability analysis of decorative joinery for furniture. ACM Trans. Graph. (TOG) 36(2) (2017). (Presented at SIGGRAPH 2017)

12. Fu, C.-W., Song, P., Yan, X., Yang, L.W., Jayaraman, P.K., Cohen-Or, D.: Computational interlocking furniture assembly. ACM Trans. Graph. SIGGRAPH 34(4) (2015). Article No. 91 